\title{
Epidemiological Investigation of Fatty Liver Disease and Abnormal Liver Function in the Republic of Kazakhstan
}

\author{
Alexander V. Nersesov ${ }^{1 *}$, Aigul M. Raissova ${ }^{2}$, Jamilya A. Kaibullayeva ${ }^{1}$, Almagul E. Jumabayeva ${ }^{1}$, \\ Mariya S. Novitskaya ${ }^{1}$ \\ ${ }^{1}$ Department of Gastroenterology and Hepatology, National Research Institute of Cardiology and Internal Diseases, Almaty, \\ Kazakhstan \\ ${ }^{2}$ Unit of Internal Diseases, National Research Institute of Cardiology and Internal Diseases, Almaty, Kazakhstan \\ Email: *alexander.nersesov@gmail.com
}

How to cite this paper: Nersesov, A.V., Raissova, A.M., Kaibullayeva, J.A., Jumabayeva, A.E. and Novitskaya, M.S. (2019) Epidemiological Investigation of Fatty Liver Disease and Abnormal Liver Function in the Republic of Kazakhstan. Open Journal of Epidemiology, 9, 309-320. https://doi.org/10.4236/ojepi.2019.94022

Received: February 11, 2019

Accepted: November 11, 2019

Published: November 14, 2019

Copyright $\odot 2019$ by author(s) and Scientific Research Publishing Inc. This work is licensed under the Creative Commons Attribution International License (CC BY 4.0).

http://creativecommons.org/licenses/by/4.0/

(C) (i) Open Access

\begin{abstract}
Aim: Little is known about the epidemiology of fatty liver disease (FLD) among high-risk primary care subjects in Kazakhstan. We investigated the prevalence of FLD (alcoholic liver disease [ALD] and non-alcoholic fatty liver disease [NAFLD]) and abnormal liver function tests (LFTs) in high risk adults in Kazakhstan during their routine visit to the general practitioner. Methods: This multicenter, cross-sectional epidemiological study was carried out at 75 clinical sites in the Republic of Kazakhstan. The full analysis set population consisted of 5109 subjects. Results: Among type 2 diabetes mellitus (T2DM) subjects, established metabolic syndrome (MetS) and/or obese subjects, the prevalence of FLD was $30.8 \%$. LFT abnormalities were found in 53.2\% subjects with FLD and 40.0\% without FLD. FLD subjects had significantly higher levels of alanine transaminase (ALT; $p<0.001$ ), aspartate transaminase (AST; $p<0.001$ ), serum total bilirubin (STB; $p<0.001$ ), and gamma-glutamyl transpeptidase (GGT; $p=0.021$ ) compared to those without FLD. LFTs increases were significantly higher in subjects with FLD compared to those without FLD and were also significantly higher in ALD subjects compared to those with NAFLD. Conclusion: This study provides insights into the prevalence of FLD and abnormal LFTs in subjects with T2DM and/or established MetS and/or who are obese in primary care settings in Kazakhstan. These findings can help healthcare providers in Kazakhstan and elsewhere to better recognize and manage patients at risk of liver disease, which will improve clinical outcomes.
\end{abstract}

\section{Keywords}

FLD, Kazakhstan, Liver, NAFLD, Prevalence 


\section{Introduction}

The incidence of liver disease is rising throughout the world and primary care practitioners are commonly faced with the scenario of a rising incidence of abnormal liver function tests (LFTs) in patients in whom there are no clinical risks, signs or symptoms of liver disease [1] [2]. The incidence of liver disease is rising throughout the world due to alcohol consumption and a range of other lifestyle factors [1].

NAFLD occurs worldwide and is the most common liver disorder in Western industrialized countries, where the major risk factors for NAFLD (central obesity, T2DM, dyslipidemia, and MetS) are common [1] [3] [4]. With a prevalence of $14 \%-34 \%$, NAFLD is recognized as the most common cause of hepatic dysfunction in the general population [1] [5] [6]. NAFLD comprises a histopathological disease spectrum from bland steatosis to non-alcoholic steatohepatitis (NASH) with significant inflammation and fibrosis, progressing to cirrhosis in approximately $20 \%$ of patients with NASH [6]. Increased body mass index (BMI), metabolic syndrome, increased fasting blood glucose, and serum triglycerides are potentially strong indicators of NAFLD [7]. Due to the indolent asymptomatic nature of NAFLD, identifying individuals with advanced disease in whom specific interventions may be required remains a clinical challenge in primary care [1]. The management of NAFLD consists of treating liver disease as well as associated metabolic co-morbidities such as obesity, hyperlipidemia, insulin resistance and T2DM [8].

Alcoholic liver disease (ALD) encompasses a broad spectrum of liver injury, ranging from simple steatosis to alcoholic hepatitis, chronic hepatitis with hepatic fibrosis, and cirrhosis [6] [9] [10]. Patients with established MetS, T2DM, overweigh/obese patients can be considered to be at high risk for FLD and development of hepatic dysfunction.

As no available official statistical data currently exists on the prevalence of FLD and abnormal LFTs (especially in terms of IHC) in the Kazakhstan population, there is a clear need for an appropriate epidemiological investigation.

More than half of Kazakhstan's population is overweight and obese [11] [12]. According to the Kazakh National Diabetes Register there are about 225,618 subjects with type 2 diabetes mellitus (T2DM) and every year the number of subjects increased by $1.8 \%$ [13]. Alcohol consumption among adults in 2005 was 6.2 liters of pure alcohol per person per year, which is relatively high (i.e. Uzbekistan: 1.8 liters) [14]. These conditions are known risk factors for developing FLD.

The awareness of general practitioners (GPs) and vigilance with regards to fatty liver disease (FLD) and hepatic dysfunction is crucial in identifying subjects with alcoholic liver disease (ALD)/NAFLD and intrahepatic cholestasis (IHC). Very little is known about the epidemiology of FLD among high-risk primary care subjects in the Republic of Kazakhstan. To the best of our knowledge, this is the largest, most detailed and comprehensive epidemiological study of FLD per- 
formed to date in Kazakhstan. It is hoped that the findings of this study help to increase the awareness of the prevalence of FLD and signs of cholestasis in patients with T2DM, obesity or metabolic syndrome (MetS), to help identify those at high risk for liver disease, and to optimize clinical management and long-term outcomes for these patients.

\section{Methods}

\subsection{Study Objectives}

The primary objective was to investigate the prevalence of FLD (i.e. ALD and NAFLD) and LFTs in a high-risk population of adults in Kazakhstan during their visit to the GP office.

The study also aimed to describe the profile of subjects in ALD/NAFLD and non-ALD/NAFLD groups, to describe abnormal LFT findings and their elevation in ALD/NAFLD and non-ALD/NAFLD. The study also sought to determine the proportion of subjects with IHC in ALD/NAFLD and non-ALD/NAFLD groups, and to make a comparison of the number of subjects with IHC based on the European Association for the Study of the Liver (EASL) criteria and simplified criteria. Finally, the study aimed to identify the risk factors for ALD/NAFLD and IHC.

\subsection{Methodology}

This was a cross-sectional (without follow-up), non-interventional, multicenter study without any investigational medicinal product or control groups, with the involvement of physicians (i.e. GPs) in outpatient clinics. Subjects attending a clinical visit, aged 18 years and older, and who fulfilled the selection criteria, were offered the opportunity to participate in the study, which was conducted between 2014-2015.

\subsection{Diagnosis and Main Criteria for Inclusion and Exclusion}

Main study inclusion criteria were; adults aged 18 years and older (male, female); provided written authorization (consent) to the physician to use and/or disclose personal and/or health data; subjects who have the following condition(s) and for whom the physician considers to check the liver function T2DM and/or established MetS and/or obese subjects $\left(B M I \geq 30 \mathrm{~kg} / \mathrm{m}^{2}\right)$. The main exclusion criteria were: subjects with known (diagnosed) cholestasis of any cause, and female subjects who were pregnant at the time of presentation to the GP. Table 1 details the FLD and co-morbidity definitions utilized in this study.

\subsection{Statistical Methods}

For variable intervals, the arithmetic mean (with 95\% CI for the mean), standard deviation (SD), and median were calculated. For ordinal variables the median value, 25- and 75-percentile were calculated. For categorical variables, frequency categories (number and \% excluding missing values) and CI for multi-category 
Table 1. FLD and co-morbidity definitions.

NAFLD diagnosis criteria

ALD diagnosis criteria

Abnormalities in LFTs

IHC based on the EASL criteria

IHC based on the simplified criteria

MetS diagnosis criteria

Pathology according to liver ultrasound
Sonographic diagnosis of fatty liver, defined as diffusely increased liver echogenicity (>right renal parenchyma) with vascular blurring;

A negative history of alcohol consumption female $>14$ units $(\mathrm{U}) /$ week, male $>21 \mathrm{U} /$ week $(1 \mathrm{U}=$ $10 \mathrm{~g}$ alcohol); and: Exclusion of liver disease of other etiology including drug-induced, autoimmune, viral hepatitis, cholestatic, metabolic, and genetic liver disease. If the 3 rd criterion cannot be rolled out it is considered as suspected for NAFLD for purpose of analysis.

The same as NAFLD, but with excessive alcohol consumption.

Deviations of LFT (ALT, AST, AP, GGT) and STB values were determined in relation to the value of ULN for each study site. The increase of at least one unit from ULN taking with reference values for each site was defined as "deviation". For ALT and AST, the deviation up to one ULN, 1 - 5 ULN have been calculated. For AP, GGT and STB, elevation by 1.5, $1.5-2$; >2 fold from ULN have been calculated.

Elevated AP levels higher than 1.5 ULN and GGT levels $>3$ ULN with or without clinically present symptoms or signs with excluded extrahepatic cholestasis at least via abdominal US.

Any elevation of AP and GGT; with or without elevation of STB; with or without clinically present symptoms or signs with excluded extrahepatic cholestasis at least via abdominal US.

Central (abdominal) obesity with waist circumference $>94 \mathrm{~cm}$ in men and $>80 \mathrm{~cm}$ in women, plus any two of the following additional criteria:
Arterial hypertension

Reduced high-density lipoprotein (HDL)-cholesterol

Raised low-density lipoprotein (LDL) - cholesterol

Raised fasting plasma glucose (FPG)

Glucose intolerance
Raised triglycerides
Blood pressure (BP) $\geq 130 / 85 \mathrm{mmHg}$

$\geq 1.7 \mathrm{mmol} / \mathrm{L}$

$<1.0 \mathrm{mmol} / \mathrm{L}$ in men; $<1.2 \mathrm{mmol} / \mathrm{L}$ in women

$\geq 3.0 \mathrm{mmol} / \mathrm{L}$

$\geq 6.1 \mathrm{mmol} / \mathrm{L}$

2-h plasma glucose during an oral glucose tolerance test is $\geq 7.8$ but $\leq 11.1 \mathrm{mmol} / \mathrm{L}$
This was established if at least one of the following was present: increased echogenicity, steatosis or diffuse changes of the liver.

variable frequency (95\% CI according to the method Sison and Glaz) were calculated. Study variables were calculated from the corresponding population without missing data. A comparison of the number of patients with IHC based on the EASL criteria and with IHC based on the simplified criteria, as well as other categorical variables was performed using the 2-sided Chi-square test. A comparison of quantitative variables was performed using the 2-sided t-test for independent samples. To assess risk factors for ALD/NAFLD and IHC, multivariate analysis of binary logistic regression, with the inclusion as independent factors and covariates of demographic, anthropometric indices, and the characteristics of a pathological condition (such as age, waist circumference, BMI) was performed.

\section{Results}

The study enrolled 5391 subjects. Following exclusions, the full analysis set (FAS) consisted of 5109 subjects. The FAS population consisted of 5109 subjects 
corresponding to the inclusion/exclusion criteria (overall population). Overall, $41.3 \%$ (2039) were male and 58.7\% (2898) were female. Full demographic and baseline characteristics are presented in Table 2 .

Details of the overall population with FLD (ALD/NAFLD) are presented in Table 3. FLD was present in 1572 (30.8\%) subjects: 1517 (29.7\%) subjects in the Total NAFLD group and 55 (1.1\%) subjects had ALD. FLD was absent in 3529 (69.1\%) of subjects. In subjects with FLD, 44.9\% (684/1525) were male and $55.1 \%(841 / 1525)$ were female. The mean $( \pm S D)$ age of subjects was $54.9 \pm 12.6$ years in the overall population.

Table 4 provides details of subjects with deviations in the biochemical analysis. LFT abnormalities were found in 2251 (44.1\%) subjects in the overall population, in $837(53.2 \%)$ subjects with FLD $(\mathrm{n}=1572)$ and in $1410(40.0 \%)$ subjects without FLD ( $\mathrm{n}=3529)$. In the FLD group the proportion of subjects with abnormalities in biochemical parameters was statistically significantly higher compared to the group without FLD: $30.0 \%$ vs $20.5 \%(p<0.001)$ for serum total bilirubin (STB); $23.0 \%$ vs $11.7 \%$ ( $p<0.001$ ) for alkaline phosphatase (AP); $37.2 \%$ vs $28.3 \%$ ( $p<0.001)$ for gamma glutamyl transpeptidase (GGT); $39.6 \%$ vs $27.1 \%$ $(p<0.001)$ for alanine transaminase (ALT); and $30.2 \%$ vs $23.2 \%(p<0.001)$ for

Table 2. Demographic and baseline characteristics (race, gender).

\begin{tabular}{|c|c|c|c|c|c|c|c|}
\hline \multicolumn{8}{|c|}{ Non-Alcoholic/Alcoholic Fatty Liver Disease } \\
\hline & & $\begin{array}{c}\text { Total } \\
\text { NAFLD }\end{array}$ & $\begin{array}{l}\text { Total } \\
\text { ALD }\end{array}$ & All FLD & No FLD & Missing & Total \\
\hline \multicolumn{8}{|c|}{ Race } \\
\hline \multirow[t]{2}{*}{ White } & Count & 601 & 15 & 22 & 623 & 1254 & 1881 \\
\hline & Valid \% & $40.4 \%$ & $42.9 \%$ & $40.7 \%$ & $40.4 \%$ & $36.9 \%$ & $38.0 \%$ \\
\hline \multirow[t]{2}{*}{ Asian } & Count & 879 & 20 & 32 & 911 & 2,112 & 3027 \\
\hline & Valid \% & $59.1 \%$ & $57.1 \%$ & $59.3 \%$ & $59.1 \%$ & $62.2 \%$ & $61.2 \%$ \\
\hline \multirow[t]{2}{*}{ Black } & Count & 2 & 0 & 0 & 2 & 4 & 6 \\
\hline & Valid \% & $0.1 \%$ & $0.0 \%$ & $0.0 \%$ & $0.1 \%$ & $0.1 \%$ & $0.1 \%$ \\
\hline \multirow[t]{2}{*}{ Other } & Count & 6 & 0 & 0 & 6 & 26 & 32 \\
\hline & Valid \% & $0.4 \%$ & $0.0 \%$ & $0.0 \%$ & $0.4 \%$ & $0.8 \%$ & $0.6 \%$ \\
\hline Valid N & Count & 1488 & 35 & 54 & 1542 & 3396 & 4946 \\
\hline Missing & Count & 29 & 1 & 1 & 30 & 133 & 163 \\
\hline Total & Count & 1517 & 36 & 55 & 1572 & 3529 & 5109 \\
\hline \multicolumn{8}{|c|}{ Gender } \\
\hline \multirow[t]{2}{*}{ Female } & Count & 834 & 7 & 7 & 841 & 2,053 & 2898 \\
\hline & Valid \% & $56.7 \%$ & $20.0 \%$ & $13.0 \%$ & $55.1 \%$ & $60.3 \%$ & $58.7 \%$ \\
\hline \multirow[t]{2}{*}{ Male } & Count & 637 & 28 & 47 & 684 & 1351 & 2039 \\
\hline & Valid \% & $43.3 \%$ & $80.0 \%$ & $87.0 \%$ & $44.9 \%$ & $39.7 \%$ & $41.3 \%$ \\
\hline Valid N & Count & 1471 & 35 & 54 & 1525 & 3404 & 4937 \\
\hline Missing & Count & 46 & 1 & 1 & 47 & 125 & 172 \\
\hline Total & Count & 1517 & 36 & 55 & 1572 & 3529 & 5109 \\
\hline
\end{tabular}


Table 3. Patients with FLD (ALD/NAFLD): overall population.

\begin{tabular}{|c|c|c|c|c|}
\hline & & \multirow{2}{*}{$\begin{array}{c}\begin{array}{c}\text { Group } \\
\text { Population }\end{array} \\
\text { ITT }\end{array}$} & \multicolumn{2}{|c|}{$95 \% \mathrm{CI}$} \\
\hline & & & $\begin{array}{c}\text { LOWER (SG) } \\
\text { CI\% }\end{array}$ & $\begin{array}{c}\text { UPPER (SG) } \\
\text { CI\% }\end{array}$ \\
\hline \multirow{6}{*}{$\begin{array}{l}\text { Non-alcoholic/ } \\
\text { alcoholic fatty } \\
\text { liver disease }\end{array}$} & Missing & $\begin{array}{c}8 \\
0.2 \%\end{array}$ & - & - \\
\hline & $\begin{array}{l}\text { Total NAFLD } \\
\text { (NAFLD } \\
\text { and suspect to } \\
\text { NAFLD) }\end{array}$ & $\begin{array}{c}1517 \\
29.7 \% \\
0.7 \%\end{array}$ & 28.4 & 31.0 \\
\hline & $\begin{array}{l}\text { Total ALD (ALD } \\
\text { and suspect to ALD) }\end{array}$ & $\begin{array}{c}55 \\
1.1 \%\end{array}$ & 67.8 & 70.4 \\
\hline & All FLD & $\begin{array}{r}1572 \\
30.8 \%\end{array}$ & 29.5 & 32.1 \\
\hline & No FLD & $\begin{array}{l}3529 \\
69.1 \%\end{array}$ & 67.8 & 70.3 \\
\hline & Total & $\begin{array}{c}5109 \\
100.0 \%\end{array}$ & - & - \\
\hline
\end{tabular}

SG: Sison and Glaz.

aspartate transaminase (AST). The proportion of Total ALD subjects with abnormalities in biochemical parameters was statistically significantly higher compared to the group of Total NAFLD: $53.7 \%$ vs $29.3 \%(p=0.001)$ for STB; $39.4 \%$ vs $22.4 \%(p=0.022)$ for AP; $60.7 \%$ vs $36.4 \%$ ( $p=0.009)$ for GGT; $57.8 \%$ vs $39.0 \%$ ( $p=0.011)$ for ALT; and $50.0 \%$ vs $29.6 \%$ ( $p=0.003)$ for AST.

Alcohol consumption was "yes" in $34.9 \%$ subjects (1749/5008) in the overall population, 33.9\% in those with FLD (518), and 35.2\% (1223) in those without FLD; $31.4 \%$ in the Total NAFLD group (463/1475) and $100.0 \%$ in the Total ALD group (55/55). The majority of subjects $(\sim 65 \%)$ consumed $\leq 1$ alcoholic drink per day. Only $9.7 \%$ (153) of subjects consumed alcohol in excessive amounts (defined as $\geq 3$ drinks per day), of them ALD were considered in 55 (35.9\%) subjects.

T2DM was diagnosed in $41.2 \%$ subjects $(2071 / 5022)$ in the overall population; 40.5\% (629/1554) subjects in FLD subjects and in 41.6\% (1441/3460) of without FLD subjects; $41.3 \%(620 / 1500)$ in the Total NAFLD group and $16.7 \%(9 / 54)$ in the Total ALD group. Obesity (BMI $\geq 30 \mathrm{~kg} / \mathrm{m}^{2}$ ) was present in $65.9 \%$ of subjects (3312/5028) in the overall population; $74.6 \%$ (1153/1545) in the group of all FLD and in $61.9 \%(2152 / 3475)$ in the group of subjects without FLD; $75.0 \%$ $(1117 / 1490)$ in the Total NAFLD group and 65.5\% (36/55) in the Total ALD group. MetS was diagnosed in $78.4 \%$ of subjects (3759/4794) in the overall population; 81.8\% (1207/1476) in FLD subjects and 76.9\% (2544/3310) in without FLD subjects; $82.1 \%(1173 / 1517)$ in the Total NAFLD group and $72.3 \%(34 / 55)$ in the Total ALD group. 
Table 4. Patients with deviations in biochemical analysis.

\begin{tabular}{|c|c|c|c|c|c|c|c|}
\hline \multicolumn{2}{|c|}{ Deviation from normal level } & $\begin{array}{c}\text { Total } \\
\text { NAFLD }\end{array}$ & $\begin{array}{l}\text { Total } \\
\text { ALD }\end{array}$ & All FLD & No FLD & Missing & Total \\
\hline \multirow{5}{*}{$\begin{array}{l}\text { Serum Total } \\
\text { Bilirubin }\end{array}$} & Count & 399 & 22 & 421 & 612 & 1 & 1034 \\
\hline & Valid \% & $29.3 \%$ & $53.7 \%$ & $30.0 \%$ & $20.5 \%$ & $12.5 \%$ & $23.5 \%$ \\
\hline & Valid N & 1362 & 41 & 1403 & 2990 & 8 & 4401 \\
\hline & $\begin{array}{c}\text { Lower (SG) } \\
\text { CI\% }\end{array}$ & 26.9 & 39.0 & 27.6 & 19.0 & - & 22.2 \\
\hline & $\begin{array}{c}\text { Upper (SG) } \\
\text { CI\% }\end{array}$ & 31.8 & 68.8 & 32.5 & 21.9 & - & 24.8 \\
\hline \multirow{5}{*}{$\begin{array}{c}\text { Alkaline } \\
\text { Phosphatase }\end{array}$} & Count & 214 & 13 & 227 & 193 & 3 & 423 \\
\hline & Valid \% & $22.4 \%$ & $39.4 \%$ & $23.0 \%$ & $11.7 \%$ & $37.5 \%$ & $16.0 \%$ \\
\hline & Valid N & 956 & 33 & 989 & 1646 & 8 & 2643 \\
\hline & $\begin{array}{c}\text { Lower (SG) } \\
\text { CI\% }\end{array}$ & 19.8 & 24.2 & 20.4 & 10.3 & - & 14.6 \\
\hline & $\begin{array}{c}\text { Upper (SG) } \\
\text { CI\% }\end{array}$ & 25.1 & 56.4 & 25.7 & 13.3 & - & 17.4 \\
\hline \multirow{5}{*}{$\begin{array}{c}\gamma \text {-Glutamyl } \\
\text { Transpeptidase }\end{array}$} & Count & 303 & 17 & 320 & 396 & 4 & 720 \\
\hline & Valid \% & $36.4 \%$ & $60.7 \%$ & $37.2 \%$ & $28.3 \%$ & $57.1 \%$ & $31.8 \%$ \\
\hline & Valid N & 833 & 28 & 861 & 1399 & 7 & 2,267 \\
\hline & $\begin{array}{c}\text { Lower (SG) } \\
\text { CI\% }\end{array}$ & 33.0 & 46.4 & 33.9 & 25.9 & - & 29.8 \\
\hline & $\begin{array}{c}\text { Upper (SG) } \\
\text { CI\% }\end{array}$ & 39.7 & 80.9 & 40.6 & 30.8 & - & 33.8 \\
\hline \multirow{5}{*}{$\begin{array}{c}\text { Alanine } \\
\text { Aminotransferase }\end{array}$} & Count & 540 & 26 & 566 & 820 & 1 & 1387 \\
\hline & Valid \% & $39.0 \%$ & $57.8 \%$ & $39.6 \%$ & $27.1 \%$ & $12.5 \%$ & $31.1 \%$ \\
\hline & Valid N & 1384 & 45 & 1429 & 3029 & 8 & 4466 \\
\hline & $\begin{array}{c}\text { Lower (SG) } \\
\text { CI\% }\end{array}$ & 36.4 & 44.4 & 37.0 & 25.5 & - & 29.7 \\
\hline & $\begin{array}{c}\text { Upper (SG) } \\
\text { CI\% }\end{array}$ & 41.8 & 72.8 & 42.3 & 28.7 & - & 32.5 \\
\hline \multirow{5}{*}{$\begin{array}{c}\text { Aspartate } \\
\text { Aminotransferase }\end{array}$} & Count & 409 & 23 & 432 & 704 & 1 & 1137 \\
\hline & Valid \% & $29.6 \%$ & $50.0 \%$ & $30.2 \%$ & $23.2 \%$ & $12.5 \%$ & $25.5 \%$ \\
\hline & Valid N & 1384 & 46 & 1430 & 3028 & 8 & 4466 \\
\hline & $\begin{array}{l}\text { Lower (SG) } \\
\quad \mathrm{CI} \%\end{array}$ & 27.2 & 37.0 & 27.8 & 21.7 & - & 24.2 \\
\hline & $\begin{array}{c}\text { Upper (SG) } \\
\text { CI\% }\end{array}$ & 32.1 & 65.5 & 32.7 & 24.8 & - & 26.8 \\
\hline \multirow{3}{*}{$\begin{array}{l}\text { Laboratory } \\
\text { Deviations }\end{array}$} & Count & 804 & 33 & 837 & 1410 & 4 & 2251 \\
\hline & Valid \% & $53.0 \%$ & $60.0 \%$ & $53.2 \%$ & $40.0 \%$ & $50.0 \%$ & $44.1 \%$ \\
\hline & Valid N & 1517 & 55 & 1572 & 3529 & 8 & 5109 \\
\hline
\end{tabular}

FLD subjects had statistically significantly higher levels of ALT $(p<0.001)$, AST $(p<0.001)$, STB $(p<0.001)$, and GGT $(p=0.021)$ compared to those without FLD. Increases in all LFTs was statistically significantly higher in subjects with FLD compared to those without FLD: AP $(p<0.001)$, ALT $(p<0.001)$, AST $(p<0.001)$, STB $(p<0.001)$, and GGT $(p<0.001)$, and in Total ALD sub- 
jects compared to Total NAFLD subjects: AP $(p<0.001)$, AST $(p=0.015)$, STB $(p=0.004)$, GGT $(p=0.005)$. Increases in all LFTs were statistically significantly higher in subjects with ALD compared to those with NAFLD: AP $(p<0.001)$, $\operatorname{ALT}(\mathrm{p}=0.014)$, AST $(p=0.012)$, STB $(p=0.006)$, and GGT $(p=0.025)$.

\section{Comparisons between Groups}

Subjects with FLD when compared to subjects without FLD had statistically significantly higher levels of ALT $(p<0.001)$, AST $(p<0.001)$, STB $(p<0.001)$, and GGT $(p=0.021)$. The rate of increase of all LFTs was statistically significantly higher in the group of patents with FLD compared to those without FLD: AP ( $p$ $<0.001)$, ALT $(p<0.001)$, AST $(p<0.001)$, STB $(p<0.001)$, and GGT $(p<$ 0.001). The group of Total ALD compared to the subjects of Total NAFLD had statistically significant higher levels of AP $(p<0.001)$, AST $(p=0.015)$, STB $(p=$ $0.004)$, and GGT $(p=0.005)$. The rate of increase of all LFTs was statistically significantly higher in the group of patents with ALD compared to those with NAFLD: AP $(p<0.001)$, ALT $(p=0.014)$, AST $(p=0.012)$, STB $(p=0.006)$, and GGT $(p=0.025)$.

IHC based on the EASL criteria was diagnosed in only $0.6 \%$ of subjects $(12 / 2178)$ in the overall population; in $0.4 \%$ subjects (3/851) in FLD subjects (all from the Total NAFLD group [0.4\%, 3 of 822]) and in 0.7\% (9/1320) of those without FLD. No subject in the Total ALD group had IHC based on the EASL criteria. IHC based on simplified criteria was diagnosed in $11.1 \%$ of subjects (242/2178) in the overall population; in 18.1\% subjects (154/851) with FLD and in $6.7 \%(881 / 320)$ without FLD; in $17.5 \%(144 / 822)$ in the Total NAFLD group and in $34.5 \%(10 / 29)$ in the Total ALD group. The number of subjects with IHC diagnosed based on the EASL criteria and the number of subjects with IHC diagnosed based on simplified criteria were statistically different $(p<0.001)$. Pathology according to liver ultrasound was found in $75.0 \%$ (9/12) of subjects with IHC diagnosed based on EASL criteria and in 97.1\% (235/242) of subjects with IHC diagnosed based on simplified criteria. In the overall population, liver pathology at ultrasound was found in $95.4 \%$ subjects (4875/5109); in 100.0\% (1572) of all FLD subjects and in 93.4\% of subjects (3295/3529) without FLD.

Binary logistic regression was developed for determination of possible risk factors of ALD, NAFLD and IHC. The following predictors were determined:

\section{Positive predictors of NAFLD}

- Increased waist circumference $(p<0.001$; odds ratio [OR] $=1.010 ; 95 \%$ confidence interval $[\mathrm{CI}]: 1.005-1.016)$;

- Increased BMI ( $p<0.001$; OR = 1.037; 95\% CI: 1.022 - 1.052);

- Presence of metabolic syndrome $(p=0.015$; OR: 1.232 ; $95 \%$ CI: 1.041 1.458).

\section{Negative predictors of ALD}

- Normal AP level ( $p=0.003$; OR: 0.011; 95\% CI: 0.001 - 0.209);

- Increase in AP level up to 1.5 upper limit of normal (ULN) $(p=0.015$; OR = 0.023; 95\% CI: $0.001-0.481$ ). 


\section{Positive predictor of IHC}

- Gender (male) $(p<0.001 ;$ OR = 1.780; 95\% CI: 1.310 - 2.417);

- Increased waist circumference was a negative predictor of IHC based on the simplified criteria $(p<0.001$; OR $=0.967 ; 95 \%$ CI: $0.954-0.981)$.

Positive predictors of IHC

- Increased BMI $(p<0.001$; OR = 1.092; 95\% CI: 1.056 - 1.129);

- Metabolic syndrome $(p<0.001 ; \mathrm{OR}=2.277 ; 95 \% \mathrm{CI}: 1.471$ - 3.527);

- Fatigue ( $p=0.031 ; \mathrm{OR}=1.805 ; 95 \%$ CI: 1.054 - 3.089).

\section{Discussion}

This epidemiological investigation helps address the paucity of official statistical data available on the prevalence of FLD and abnormal LFTs (especially in terms of IHC) in the Kazakhstan population. The study provides considerable insight into the prevalence of FLD, NAFLD, ALD, and abnormal LFTs in subjects with T2DM and/or established MetS and/or obese in a primary care setting in Kazakhstan. It demonstrates the necessity to screen high-risk subjects for FLD at the GP level; this includes subjects with evidence of insulin resistance, MetS and metabolic risk factors, such as obesity.

The rate of any FLD in this population was 30.8\% (of NAFLD 29.7\%; of ALD $1.1 \%)$. The rate of any abnormalities in LFTs was $44.1 \%$; it was higher those with FLD (53.2\%) and lower in those without FLD (40.0\%).

Demographic data were similar between study groups, except for gender distribution. In total ALD subjects, $87.0 \%$ were male. The majority of subjects (about $65 \%$ ) consumed $\leq 1$ alcoholic drink per day. Only $9.7 \%$ of subjects consumed alcohol in excessive amounts (defined as $\geq 3$ drinks per day); of these subjects, ALD was considered in $36 \%$.

Evaluation of LFT abnormalities showed that subjects with ALD were characterized by the highest level of biochemical parameters. Normal biochemical parameters (less than upper limit of normal ranges) varied from $7.7 \%$ to $40.0 \%$ in this group, whereas in the overall population $68.2 \%$ to $84.0 \%$ of subjects had normal biochemical parameters.

IHC diagnosed based on EASL criteria were found to be quite rare with only $0.6 \%$ of subjects identified. Interestingly, the number of subjects with IHC diagnosed based on EASL or simplified criteria were significantly different. IHC diagnosed based on simplified criteria was found in $11.1 \%$ subjects; this proportion was higher in FLD subjects (18.1\%) and lower in those without FLD (6.7\%). Most subjects with IHC based on EASL (75.0\%) and simplified criteria (97.1\%) had pathology demonstrated by liver ultrasound.

Possible risk factors for NAFLD, ALD or IHC were determined using binary logistic regression. Predictors for IHC diagnosed based on simplified criteria were male gender, waist circumference, BMI, MetS, and fatigue (as a symptom of IHC). Models of logistic regression for IHC based on EASL criteria could not be developed properly due to a small number of cases included in the analysis.

The principle finding of this study was that among Kazakh adult subjects with 
T2DM and/or established MetS and/or obese subjects who routinely attended outpatient clinics, the prevalence of FLD (NAFLD and ALD) was $30.8 \% ; 29.7 \%$ of subjects were considered as having NAFLD and $1.1 \%$ of subjects as having ALD. It is interesting to compare our results with other published studies. Recent studies report the prevalence of NAFLD in the region of $14 \%-34 \%$ of the general-population in Europe, Asia, and America [1] [5] [6]. A World Health Organization (WHO) assessment in 2002 reported 4\% of the burden of diseases and $3.2 \%$ of all deaths globally were attributable to alcohol [15]. In addition, ALD was considered the foremost health risk in developing countries and ranks third in developed countries [16]. Liver disease prevalence results reported here for this Kazakh population are similar to those reported in other global regions; although the level of ALD may be lower than anticipated, possibly due to study limitations.

Abnormal LFTs were found in just over half of subjects with FLD and in $40.0 \%$ of those without FLD. The demographic and clinical profiles of the subjects were typical for subjects with MetS, obesity and other risk factors for FLD, and reflect the nature of each disease (NAFLD, ALD, absence of FLD). Abnormal LFTs were more common in subjects within the FLD group than in subjects without FLD; elevation of AP was found twice as often, elevation of ALT and SBT was 1.5 times more often, and elevation of AST and GGT was 1.3 times more often. The majority of subjects in both FLD and non-FLD groups had increased LTFs by 1.5 ULN. The proportion of subjects with abnormalities in biochemical parameters was statistically significantly higher in the FLD group compared to those without FLD, and similarly, was statistically significantly higher in the Total ALD group compared to those in the Total NAFLD group.

It is interesting to note that in primary care settings, ALT, AST, and STB are used almost twice as often as GGT and AP for assessment of liver function. Data were available for STB in $86.1 \%$ of subjects whereas data for GGT was available in just $44.4 \%$.

IHC was more frequently diagnosed based on simplified criteria than based on the EASL criteria. It should be noted that the EASL criteria for IHC are mostly applicable for primary cholestatic diseases and, probably, not appropriate for secondary IHC diseases such as NAFLD or ALD. Possible risk factors were determined for NAFLD, ALD and IHC; many of them are related to MetS. IHC was also associated with male gender and fatigue. Ultrasonography signs of liver pathology were found in $95.4 \%$ of enrolled subjects.

The main limitation of our study may be the small number of valid cases in particular study groups, most notably in the number of subjects identified as having ALD and subjects with IHC. All study variables were analyzed using valid cases, without replacing missing data, and the valid percent was calculated. In addition, due to the observational design, many data were absent for some variables (up to $50 \%$ of the sample size); therefore, such data should be interpreted with caution.

The present study strongly suggests that at-risk subjects should undergo di- 
agnostic procedures for the diagnosis of NAFLD and in subjects with features of MetS. Screening for NAFLD by liver enzymes and/or ultrasound should be part of routine work-up [17]. It is hoped that this study will enable healthcare providers in Kazakhstan to better recognize and manage subjects at risk of liver disease, which will result in improved clinical outcomes.

\section{Acknowledgements}

Writing assistance was funded by Abbott Products Operations AG and was provided by Martin Guppy.

\section{Funding}

The study was funded by Abbott Kazakhstan LLP.

\section{Ethical Approval}

All procedures performed in studies involving human participants were in accordance with the ethical standards of the institutional and/or national research committee and with the 1964 Helsinki declaration and its later amendments or comparable ethical standards. Informed consent was obtained from all individual participants included in the study.

\section{Conflicts of Interest}

A. N. has received personal fees from Abbott Laboratories (as the advisory expert of the study). The other authors disclose no conflicts.

\section{References}

[1] Armstrong, M.J., Houlihan, D.D., Bentham, L., Shaw, J.C., Cramb, R., Olliff, S., et al. (2012) Presence and Severity of Non-Alcoholic Fatty Liver Disease in a Large Prospective Primary Care Cohort. Journal of Hepatology, 56, 234-240. https://doi.org/10.1016/j.jhep.2011.03.020

[2] Fan J.G. (2013) Epidemiology of Alcoholic and Nonalcoholic Fatty Liver Disease in China. Journal of Gastroenterology and Hepatology, 28, 11-17. https://doi.org/10.1111/jgh.12036

[3] Paschos, P. and Paletas, K. (2009) Non-Alcoholic Fatty Liver Disease and Metabolic Syndrome. Hippokratia, 13, 9-19.

[4] Younossi, Z.M., Stepanova, M., Afendy, M., Fang, Y., Younossi, Y., Mir, H., et al. (2011) Changes in the Prevalence of the Most Common Causes of Chronic Liver Diseases in the United States from 1988 to 2008. Clinical Gastroenterology and Hepatology, 9, 524-530.e1. https://doi.org/10.1016/j.cgh.2011.03.020

[5] Bayard, M., Holt, J. and Boroughs, E. (2006) Nonalcoholic Fatty Liver Disease. American Family Physician, 73, 1961-1968.

[6] Jüngst, C., Berg, T., Cheng, J., Green, R.M., Jia, J., Mason, A.L., et al. (2013) Intrahepatic Cholestasis in Common Chronic Liver Diseases. European Journal of Clinical Investigation, 43, 1069-1083. https://doi.org/10.1111/eci.12128

[7] Anurag, L., Aniket, S., Shalik, J., Amarja, L., Dhananjay, R. and Sachin, J. (2015) Non-Alcoholic Fatty Liver Disease Prevalence and Associated Risk Factors-A Study 
from Rural Sector of Maharashtra. Tropical Gastroenterology, 36, 25-30. https://doi.org/10.7869/tg.241

[8] Onyekwere, C.A., Ogbera, A.O., Samaila, A.A., Balogun, B.O. and Abdulkareem, F.B. (2015) Nonalcoholic Fatty Liver Disease: Synopsis of Current Developments. Nigerian Journal of Clinical Practice, 18, 703-712. https://doi.org/10.4103/1119-3077.163288

[9] Ishii, H., Horie, Y., Yamagishi, Y. and Ebinuma, H. (2010) Alcoholic Liver Disease and Its Relationship with Metabolic Syndrome. Japanese Medical Association Journal, 53, 236-242.

[10] Chiang, D.J. and McCullough, A.J. (2014) The Impact of Obesity and Metabolic Syndrome on Alcoholic Liver Disease. Clinical Liver Disease, 18, 157-163. https://doi.org/10.1016/j.cld.2013.09.006

[11] Kadyrova, R.Kh. and Salkhanov, B.A. (1990) The Prevalence of Obesity among the Adult Population of Kazakhstan. Voprosy Pitaniia, 1, 30-33.

[12] Salkhanov, B.A. and Kadyrova, R.Kh. (1989) The Role of Nutritional Factors in the Development of Obesity among the Adult Residents of Kazakhstan. Voprosy Pitaniia, 4, 32-36.

[13] Sikhayeva, N., Talzhanov, Y., Iskakova, A., Dzharmukhanov, J., Nugmanova, R., Zholdybaeva, E. and Ramanculov, E. (2018) Type 2 Diabetes Mellitus: Distribution of Genetic Markers in Kazakh Population. Clinical Interventions in Aging, 13, 377-388. https://doi.org/10.2147/CIA.S156044

[14] World Health Organization (2016) Kazakhstan Statistics Summary (2002-Present). http://apps.who.int/gho/data/node.country.country-KAZ

[15] World Health Organization (2002) The World Health Report 2002-Reducing Risks, Promoting Healthy Life. http://www.who.int/whr/2002/en/whr02_en.pdf?ua=1

[16] Kim, W.R., Brown Jr., R.S., Terrault, N.A. and El-Serag, H. (2002) Burden of Liver Disease in the United States: Summary of a Workshop. Hepatology, 36, 227-242. https://doi.org/10.1053/jhep.2002.34734

[17] European Association for the Study of the Liver (EASL), European Association for the Study of Diabetes (EASD), European Association for the Study of Obesity (EASO) (2016) EASL-EASD-EASO Clinical Practice Guidelines for the Management of Non-Alcoholic Fatty Liver Disease. Obesity Facts, 9, 65-90.

https://doi.org/10.1159/000443344 